\title{
Omental Pedicled Flap for Pulmonary Tuberculosis Sequelae
}

Wamalwa Alex Okello, Nangole Ferdinand Wanjala

School of Medicine, University of Nairobi

Correspondence to: Dr. Wamalwa Alex, P.O. Box 2212- 00202, Nairobi. Kenya. Email: aleoke@gmail.com

\section{Summary}

The omental flap should be prophylactically used in post-pneumonectomy bronchial stump reinforcement where the underlying chronic inflammatory condition poses high risk for bronchial dehiscence. We present a unique case of pulmonary tuberculosis (TB) complicated by empyema, chronic pulmonary aspergillosis and extensive pulmonary destruction that necessitated a pneumonectomy. The patient developed a broncho-atmospheric fistula. Despite initial closure of

\section{Introduction}

Since first described for patching a perforated peptic ulcer disease, the further developed omental pedicled flap has been successfully reported in closure of post pneumonectomy bronchopleural fistula (BPF) (1). Up to $20 \%$ of patients undergoing pneumonectomy develop $\mathrm{BPF}$; more so on the right due to extensive dissection undertaken (2). Patients with tuberculosis (TB) are prone to poor wound healing. We present a case of post TB sequelae requiring pneumonectomy and closure of resulting BPF with the omental pedicled flap

\section{Case Presentation}

We present a case of a 29-year-old Human Immunodeficiency Virus (HIV) negative patient who had been diagnosed and managed for sputum smear positive pulmonary tuberculosis (TB) at a peripheral facility. During his standard six-month treatment regime, a right tube thoracostomy was inserted to alleviate severe dyspnea due to pleural effusion. On completion, the repeat sputum smear was negative (3).

However, severe episodes of dyspnea due to recurrence of pleural effusion (relaxation atelectasis) negated removal of the tube thoracostomy. He presented to the cardiothoracic surgeon 1 year later with empyema the resulting bronchopleural fistula with an omental pedicled flap; subsequent complications highlight the missed opportunity at preventing its occurrence.

Key words: Prophylactic, Omental Flap, Tuberculosis, Pneumonectomy

Ann Afr Surg. 2017; 14(2):108-112

DOI:http://dx.doi.org/10.4314/aas.v14i2.12

(C) 2017 Author. This work is licensed under the Creative Commons Attribution 4.0 International License.

thoracis and a decortication and wash out were performed via a right open thoracotomy. Postoperatively the empyema persisted and his symptoms did not abate (Figure 1). A pleural biopsy done was reported as granulomatous pleuritis positive for fungi with further morphological identification of Aspergillus spp. Ziehl Nielsen stain for acid fast bacilli was negative. A sputum sample for Xpert MTB (Mycobacterium tuberculosis complex)/RIF (Rifampicin) test was also negative.

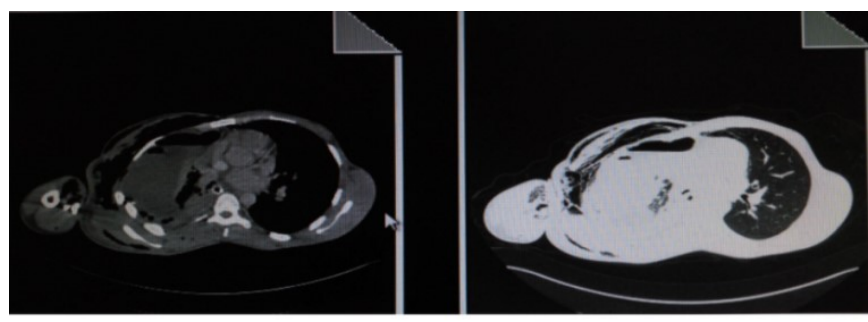

post-thoracotomy and decortication empyema thoracis associated atelectasis of remaining right lung subcutaneous emphysema of neck, upper anterio and lateral chest wall

Figure 1:Chest Radiograph and Computed Tomography (CT) Scan Illustrating Recalcitrant Empyema ThoracisPost Thoracotomy \& Decortication 
While on antifungals he developed hemoptysis, precipitating a relook right open thoracotomy where a right pneumonectomy was performed due to the extensive pulmonary destruction encountered. Immediate postoperative recovery was uneventful and symptoms resolved. The lung specimen biopsy was firm consolidated and having focal areas of hemorrhage. There was tissue pleural surface exudate; bronchiole smooth muscle hypertrophy; collapsed alveolar with mononuclear cell infiltrate and pneumocyte cell hyperplasia. During out-patient review visits (two weeks post-surgery); he was noted to have a discharging sinus at the incision site. Soon thereafter, he noted serous-purulent discharge and gush of air from a right chest wall wound especially during coughing. He had significantly lost weight (4 kilograms) despite good appetite and feeding.

A plastic surgery consultation revealed an ambulant patient, in fair general condition with noted poor nutrition and having normal vital signs. Parameters in the general examination were normal. A focused respiratory system examination revealed normal respiratory rate of 18 breathes/minute, a central trachea and the right chest wall exhibited reduced chest excursion, dullness to percussion and absent breathe sounds. There was a $1 \mathrm{~cm}$ diameter fistula on the healing right lateral thoracotomy wound (See Figure 2). Other systemic examination was non-contributory. An impression of a right post-pneumonectomy bronchoatmospheric fistula was made. His laboratory indices were all within normal ranges: normal complete blood count, normal renal function test and normal coagulation profile.A chest radiograph-PA view revealed a right hemithoraxlucency with pleural opacification at the base and costophrenic angle (Figure $3)$.

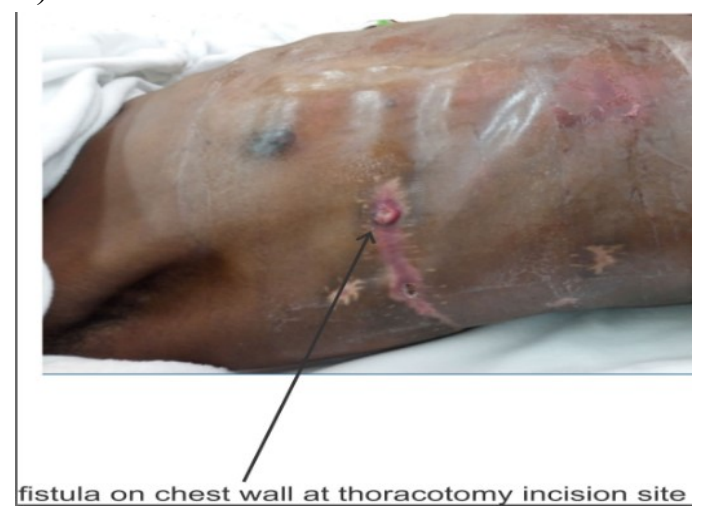

Figure 2: Chest Wall Fistula

A plan for omental flap closure of the underlying bronchopleural fistula was made.

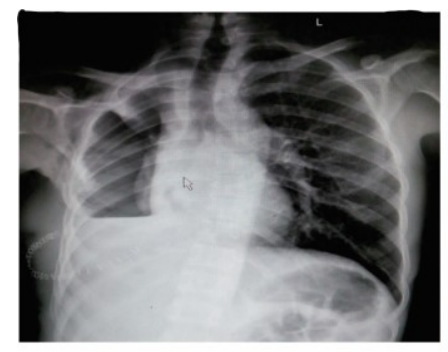

early post right pneumonectomy

Late post right pneumonectomy Increased lucency of right hemithorax

Pleural base/costophrenic angle opacification - empyema thoracis

Figure 3: Chest Radiographs Illustrating Early Vs Late Post Pneumonectomy Findings

Minimal empyema was drained on right anterolateral open thoracotomy. Thorough surgical debridement and pleural cavity irrigation was done. A pedicled omental flap based on the left gastro-epiploic artery was raised via a midline supraumbilical laparotomy incision (See Figure 4). It was delivered into the right hemithorax via a blunt dissection through the diaphragm in the right subxiphoid region. The bronchopleural fistula was identified: jetting of water was seen over the fistula site on gentle saline irrigation of the bronchial stump whilst positive tracheal ventilation was performed (See Figure 5). Since the omentum was not significantly bulky, a dual flap omental closure was performed using nonabsorbable monofilament 3-0 prolene (Figure 6). An air tight seal was confirmed: ventilatory pressures were maintained on tracheal ventilation. The fistula tract was excised and thoracotomy wound closed in layers. A tube thoracostomy was left in-situ.

Immediate postoperative period was uneventful. Pain was controlled using triple analgesia (paracetamol, tramadol and diclofenac) plus thoracic epidural. Coughing ceased and no bubbling was seen in the underwater drain canister. The tube thoracostomy was removed on the $5^{\text {th }}$ postoperative day.However, the patient developed empyema of the right hemithorax with a recurrent BPF (See Figure 7). Via video-assisted thoracoscopic surgery (VATS), a washout was done and repair of the recurrent BPF using ethibond excel 20 . Five days after the VATS his symptoms recurred and progressively worsened.

The ANNALS of AFRICAN SURGERY, July 2017 Volume 14 Issue 2 


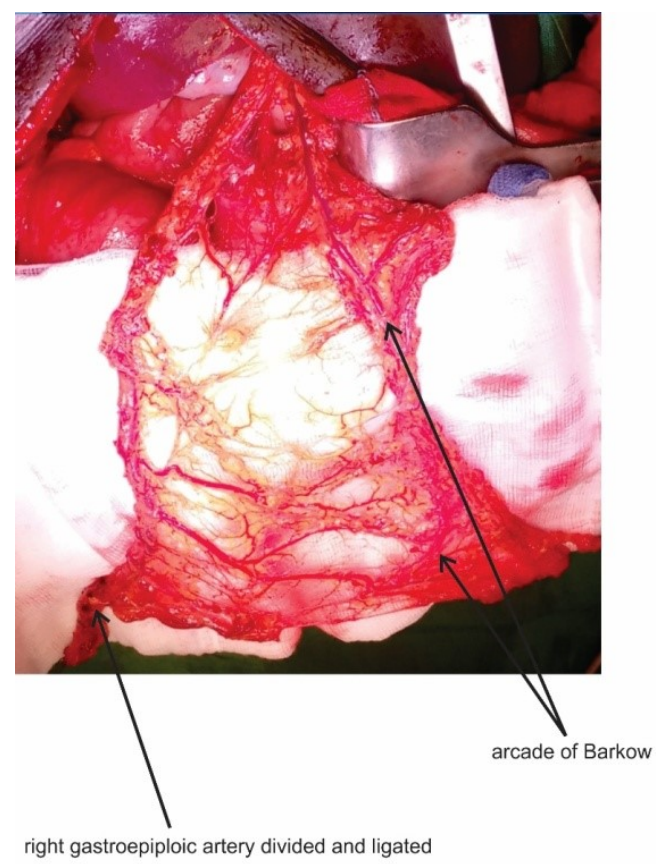

omental pedicled flap harvested- based on left gastroepiploic artery

Figure 4: OmentalPedicled Flap Harvested

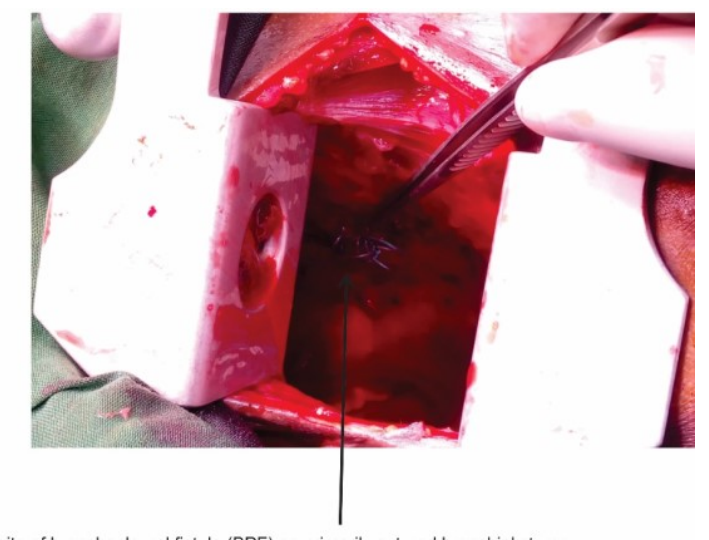

Figure 5: Site of Bronchopleural Fistula (BPF)

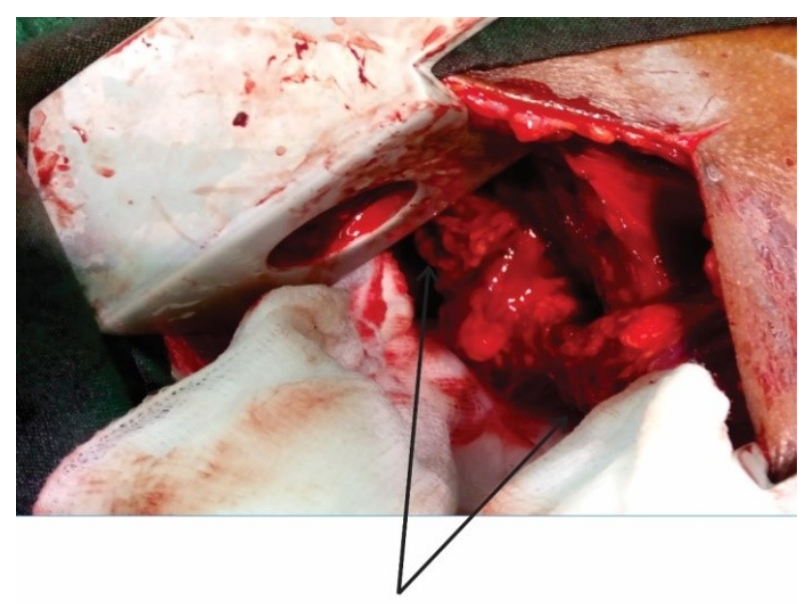

pedicled omental flap inset over bronchial stump

Figure 6: Bronchopleural Fistula (BPF) Closed With Omental Flap

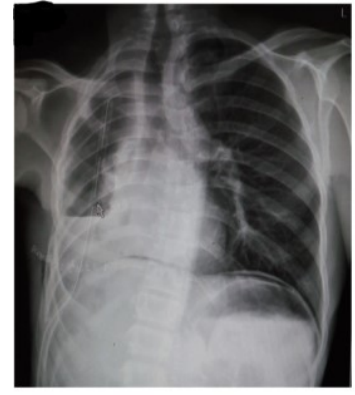

early post omental patching

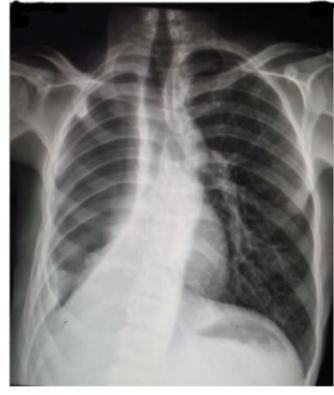

late post omental patching increased lucency of right hemithorax consistent with recurrent bronchopleural fistula
Figure 7: Chest Radiographs Illustrating Changes from Early to Late Post Omental Patching

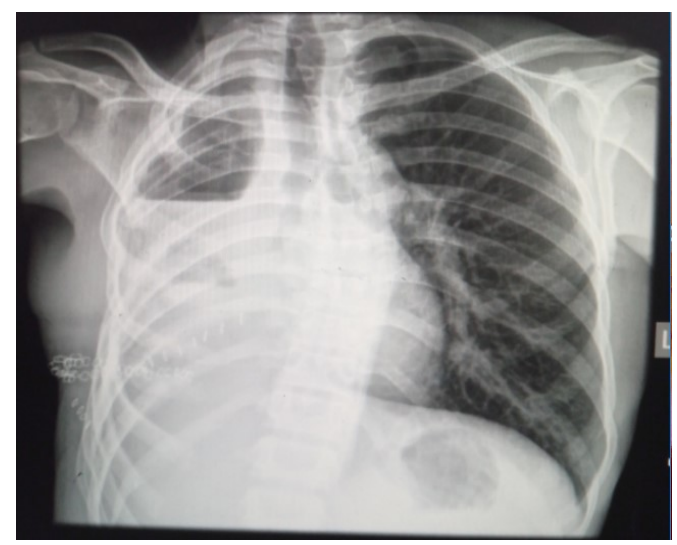

Figure 8: Chest Radiograph of 6 Months Follow-Up Post Omental Repositioning

A multidisciplinary redo thoracotomy was performed where a dislodged omental flap was repositioned over the debrided bronchial stump. There was no active pleural space infection. The patient has had an uneventful recovery so far (six months after his last surgery) and is on follow up at the cardiothoracic surgery and wound clinics

\section{Discussion}

In our set up, Kenya is among the 30 high TB burden countries globally with a TB prevalence of 558 per 100,000 people (4). Chronic pulmonary aspergillosis, due to either Aspergillus fumigatus, Aspergillusniger or Aspergillusflavus; is a poorly understood sequelae of pulmonary $\mathrm{TB}$ and affects an estimated 1.2 million people globally (5). Interesting in our case was no report of cavitating pulmonary lesion; that would have been expected as the preceding complication. With the Aspergillus spp. identified in pleural biopsy, new onset hemoptysis and intraoperative unfavorable lung; a pneumonectomy was done. Prevention is the best cure 
to post-pneumonectomy BPF and adhering to the following surgical principles is key (6):

1. Tension-free closure of a disease free short bronchial stump

2. Minimizing dissection, gentle tissue handling and precise placement of sutures

3. Peri-operative infection control

4. Optimal patient nutritional status

5. Prophylactic Bronchial stump reinforcement during pneumonectomy using pedicled pericardial fat pad, azygos vein flap, pleural flap, vascular perforator flaps or pedicled intercostal muscle flap

Indications for prophylactic bronchial stump reinforcement include: diabetes mellitus, malnutrition, advanced age ( $>$ 60years), steroid administration, perioperative radiotherapy and concurrent chemotherapy, hepatic disease (cirrhosis and hypoalbuminemia), right sided pneumonectomy, a large diameter $(>25 \mathrm{~mm})$ bronchial stump, prolonged post-operative mechanical ventilation; which are associated with higher risk of post-pneumonectomy BPF (7). Chronic inflammatory conditions like tuberculosis are however not recognized. Our assertion is that with the underlying chronic inflammatory condition, the patient was at high risk for bronchial dehiscence due to inevitable poor wound healing. Bronchial stump reinforcement should have been performed at pneumonectomy. TB complicated by pleural space infection (empyema thoracis) and chronic pulmonary aspergillosis raised all the flags to hindrance of bronchial stump healing by first intention. A multidisciplinary approach is essential. We propose use of pedicled omental flap for bronchial reinforcement in cases with underlying chronic inflammatory states. Its unique capacity to contain infective processes prefers its use to other muscle or vascular based flaps. Whereas a BPF is a sinus tract between the bronchus and pleural space, a bronchoatmospheric fistula results if there is a direct communication between the bronchus and the environment via chest wall. BPF has mortality rates of $16.4-71.2 \%$ (8). BPF can be classified according to the time of onset post-surgery (9):

1. Early BPF -1 to 7 days post-surgery

2. Intermediate BPF- 8 to 30 days post-surgery

3. Late BPF- more than 30 days post-surgery
Our patient developed an intermediate BPF with the resulting symptomatology of a broncho-atmospheric fistula. Pleural space infection (early empyema) and surgical site infection likely led to this presentation. Empyema complicates pneumonectomy in 5\% of the cases. We performed single stage pleural cavity debridement, irrigation and omentoplasty; since we had encountered minimal empyema and were confident of our debridement. We opted against open window thoracotomy procedure (Eloesser open drainage or Clagett procedure). The omental pedicle flap has key features promoting its use:

1. Malleable and easily conforms to irregular surfaces.

2. It has a long large caliber and reliable vascular pedicle-based on either right or left gastroepiploic artery.

3. Good volume - can measure up to $25 \times 35$ centimeters (10)

4. Minimal donor site morbidity and easy to harvest

5. Stimulates angiogenesis and revascularization (11, 12)

6. High absorptive capacity and able to relieve lymphedema (13)

7. Immune regulatory properties- ability to contain infective processes $(14,15)$

Our combined abdominal and thoracic approach allowed us to harvest the entire omentum and increase its arc of rotation by basing it on the left gastro-epiploic artery to cover the BPF tension-free (16). Complications associated with omental pedicled flap include: ileus, bowel obstruction, gastric outlet obstruction (especially if based on the right gastroepiploic artery), diaphragmatic herniation and omental flap necrosis.

In our case, the patient developed empyema post omental patching with a recurrent BPF. We opted for VATS as the least invasive procedure for washout and also primarily repaired the small BPF (17).

\section{Conclusion}

We advocate for prophylactic bronchial stump reinforcement using the omental pedicled flap where the underlying disease pathology hinders normal healing patterns. Our case highlights pulmonary TB and its sequelae as an underlying high risk for bronchial dehiscence. 


\section{References}

1. Nangole FW, Khainga SO, Ruturi A, et al. Dual omental flap in obliterating post-pneumonectomy bronchopleural fistulae. East Afr Med J. 2015; 92(7): 366-70

2. Darling GE, Abdurahman A, Yi QL, et al. Risk of a right pneumonectomy: role of bronchopleural fistula. Ann ThoracSurg2005; 79: 433-7

3. World Health Organization. Treatment of tuberculosis: guidelines for national programs, 4th ed. WHO/HTM.TB/2009.420. Geneva, Switzerland. World Health Organization 2009

4. Kenya Tuberculosis Prevalence Survey 2015-5016. Assessing Kenya's TB Burden. Ministry of Health. Republic of Kenya.

5. Denning DW, Pleuvry A, Cole DC. Global burden of chronic pulmonary aspergillosis as a sequel to pulmonary tuberculosis. Bull. World Health Organ. 2011; 89(12): 864-872

6. Llewellyn-Bennett R, Wotton R, West D. Prophylactic flap coverage and the incidence of bronchopleural fistulae after pneumonectomy. Interact Cardiovasc Thorac Surg 2013; 16(5):681-5.

7. Panagopoulos ND, Apostolakis E, Koletsis E, et al. Low Incidence of Bronchopleural Fistula after Pneumonectomy for Lung Cancer. Interact Cardiovasc Thorac Surg 2009; 9 (4): 571-575.

8. Hollaus PH, Lax F, El-Nashef BB Et Al. Natural History of Bronchopleural Fistula after Pneumonectomy: A Review of 96 Cases. Ann Thorac Surg 1997; 63: 1391-6

9. Varoli F, Roviaro G, Grignani F, et al. Endoscopic Treatment of Bronchopleural Fistulas. Ann Thorac Surg 1998; 65(3): 807-9

10. Das SK. The Size of the Human Omentum and Methods of Lengthening it for Transplantation. Br J Plast Surg 1976; 29(2):170-44

11. Bruzoni M, Steinberg GK, Dutta S. Laparoscopic Harvesting of Omental Pedicle Flap for Cerebral Revascularization in Children with Moyamoya Disease. J Pediatr Surg 2016; 51(4):592-7.

12. Litbarg NO, Gudehithlu KP, Sethupathi $P$, et al. Activated Omentum Becomes Rich in Factors that Promote Healing and Tissue Regeneration. Cell Tissue Res 2007; 328(3):487-497.
13. Nguyen AT, Suami H, Hanasono MM, et al. LongTerm Outcomes of the Minimally Invasive Free Vascularized Omental Lymphatic Flap for the Treatment of Lymphedema. J Surg Oncol 2017; 115(1): 84-89.

14. Miyamoto $Y$, Akiyama T, Sakamoto $Y$, et al. Omental Flap After Pelvic Exenteration for Pelvic Cancer. Surg Today 2016; 46(12):1471-1475.

15. Kitano M. Omentoplasty in Thoracic Surgery. Gen Thorac Cardiovasc Surg 2008; 56(10): 483-9.

16. Boulton BJ, Force S. The Technique of Omentum Harvest for Intrathoracic Use. Operative Techniques in Thoracic and Cardiovascular Surgery. 2010; 15 (1): 53-60.

17. Gossot D, Stern JB, Galetta D, et al. Thoracoscopic Management of Post Pneumonectomy Empyema. Ann Thorac Surg 2004; 78(1):273-6. 